\title{
Long-term oncologic outcomes in pathologic tumor response after neoadjuvant chemoradiation for locally advanced rectal cancer
}

\author{
Jeong-Soo Park, Jeong-Heum Baek, Won-Suk Lee, Jun-Young Yang, Woon-Kee Lee, Kun-Kuk Kim, Yeon-Ho Park \\ Department of Surgery, Gil Medical Center, Gachon University College of Medicine, Incheon, Korea
}

Purpose: The status of tumor regression in rectal cancer after neoadjuvant concurrent chemoradiotherapy (CCRT) has significant effect on tumor recurrence and patient survival. The aim of this study was to evaluate the long-term oncologic outcomes of rectal cancer patients presenting complete response or down-staging of rectal cancer compared to patients with non-response after neoadjuvant therapy in advanced mid-to-lower rectal cancer.

Methods: We retrospectively reviewed 79 patients with stage II/III mid-to-lower rectal cancer following neoadjuvant CCRT between March 2003 and April 2012. Patients were classified into three groups according to down-staging tumor response after neoadjuvant CCRT: complete response group (CRG), partial response group (PRG), and non-response group (NRG).

Results: Of the 79 patients in the study, eight (10.1\%), 31 (39.2\%), and 40 (50.7\%) were classified as CRG, PRG, and NRG, respectively. Median follow-up period was 57 months. There was significant difference in local recurrence $(P=0.012)$ between the three groups, yet there was no significant difference in overall survival ( $C R G, 100 \%$; $P R G, 82.5 \%$; NRG, 74.0\%; $P=0.244$ ). There was a significant difference in disease-free survival (CRG, 100\%; PRG, 90.1\%; NRG, 57.7\%; $P=0.006$ ).

Conclusion: Tumor response with complete response or down-staging provided better oncologic outcomes in terms of disease-free survival and local recurrence in locally advanced rectal cancer patients.

Keywords: Rectal neoplasms, Chemoradiotherapy, Surgical oncology

\section{INTRODUCTION}

Neoadjuvant concurrent chemoradiotherapy (CCRT) has become a standard strategy for patients with locally advanced rectal adenocarcinomas $[1,2]$. Neoadjuvant CCRT for patients with locally advanced rectal cancer was associated with lower local recurrence (LR) and higher rates of sphincter preservation $[1,3]$.

Recent studies have suggested that the complete remission following neoadjuvant CCRT is a significant prognostic factor for

Received: Apr 2, 2018 Accepted: May 30, 2018

Correspondence to: Jeong-Heum Baek

Department of Surgery, Gil Medical Center, Gachon University College of Medicine, 21 Namdong-daero 774beon-gil, Namdong-gu, Incheon 21565, Korea

Tel: +82-32-460-3241, Fax: +82-32-460-3247

E-mail: gsbaek@gilhospital.com

Copyright (C) Korean Society of Surgical Oncology

This is an Open Access article distributed under the terms of the Creative Commons Attribution Non-Commercial License (http://creativecommons.org/licenses/by-nc/4.0) which permits unrestricted non-commercial use, distribution, and reproduction in any medium, provided the original work is properly cited. survival benefit including both disease-free survival (DFS) and overall survival (OS) [4-6]. Some studies suggest that pathologic down-staging and pathologic complete response (CR) can be a signal of survival advantage $[7,8]$. The status of pathologic stage or tumor regression rate of the disease after neoadjuvant chemoradiation have significant impact on DFS $[9,10]$.

However, few studies have shown the oncologic outcomes between CR, down-staging and non-down-staging of rectal cancer after neoadjuvant CCRT in terms of recurrence and survival. The aim of this study was to evaluate the outcomes in survival of rectal cancer patients presenting CR and a down-staging tumor response following neoadjuvant CCRT in advanced mid-to-lower rectal cancer compared to patients with non-response after neoadjuvant therapy.

\section{METHODS}

A total of, 1,077 patients who underwent radical surgery for rectal cancer at Gil Medical Center between March 2003 and April 2012 was analyzed. All relevant data were entered in a prospectively 
maintained database and reviewed by the authors for verification. The inclusion criteria for this study were as follows: (1) tumor located no more than $10 \mathrm{~cm}$ from the anal verge; (2) clinical TNM stage II or III (T3-T4 or N positive and M0) on abdominopelvic computed tomography (APCT), colorectal magnetic resonance imaging (MRI), and chest computed tomography (CT); (3) patients with rectal adenocarcinoma. Patients who underwent emergent operation during neoadjuvant CCRT, had no follow-up visits, or did not undergo adjuvant chemotherapy after surgery were excluded. Seventy-nine rectal cancer patients were included in this study.

The included patients were divided into the following three groups by pathologic report after surgery: complete response group (CRG), partial response group (PRG), and non-response group (NRG) according to the status of tumor down-staging or rectal cancer after neoadjuvant CCRT. We defined the patients in PRG as patients with down-staging apart from CRG after CCRT. The stage of rectal cancer did not decline in patients in NRG.

The preoperative clinical evaluation included digital rectal exam, colonoscopy, APCT, chest CT, colorectal MRI, liver function test, and carcinoembryonic antigen (CEA), CA 19-9, and body mass in$\operatorname{dex}(\mathrm{BMI})$. The preoperative physical status of the patients was categorized according to the American Society of Anesthesiology classification. The evaluation of locoregional tumor staging (cT stage, $\mathrm{cN}$ stage) was categorized according to MRI and CT findings.

All patients received pelvic radiotherapy (RT) with concurrent chemotherapy. The concurrent chemotherapy was performed at the first and fifth week of radiation with bolus intravenous therapy of 5 -fluorouracil $400 \mathrm{mg} / \mathrm{m}^{2}$ and leucovorin $20 \mathrm{mg} / \mathrm{m}^{2}$ for 5 days per week. Oral capecitabine was prescribed at a dose of $1,650 \mathrm{mg} /$ $\mathrm{m}^{2}$ /day, divided into two doses given 12 hours apart.

All patients received external beam radiation therapy (median dose, 50.40 Gy; range, 48.4-55.8 Gy), according to the previously published techniques [11]. Pelvic RT was delivered with a 3-field technique by using a megavoltage linear accelerator (range, 6-10 MV). Surgery was attempted 6 to 8 weeks after completion of neoadjuvant chemoradiotherapy. All patients underwent low anterior resection or abdominoperineal resection and total mesorectal excision (TME). All patients were followed up postoperatively every 3 months for the first 3 years, every 6 months until 5 years and annually thereafter. A clinical examination, laboratory measurements of serum CEA and CA 19-9, and chest X-ray were performed at each follow-up. APCT, chest CT were performed annually. Recurrence was evaluated on the basis of physical, radiologic, and pathologic findings. Survival was calculated from the date of surgery to the last visit or death. All medically fit patients received adjuvant chemotherapy after surgery for 4 months according to National Comprehensive Cancer Network guidelines.
Statistical evaluation was carried out using the statistical package SPSS for Windows version 18.0 (SPSS Inc., Chicago, IL, USA). Continuous variables were expressed as means \pm standard deviation. Data between groups were analyzed with independent t-test or chi-square test and analysis of variance. Survival rates were assessed by the Kaplan-Meier method and were compared using the log-rank test. Two-tailed probability P-values less than 0.05 were considered to indicate a significant difference.

\section{RESULTS}

Table 1 shows the preoperative clinical stage and pathologic stage of the 79 patients followed by neoadjuvant CCRT and curative surgical resection. Among the 79 patients treated with neoadjuvant CCRT, the preoperative stage of disease was CT3N0M0 in 35 patients and cT3N+M0 in 44 patients. CR to neoadjuvant CCRT was pathologically confirmed in eight of 79 patients (10.1\%). Tumor down-staging occurred in 39 cases (49.3\%).

Baseline characteristics of CRG, PRG, and NRG patients are shown in Table 2. There were no significant differences in the age, gender, BMI, tumor markers, such as CEA and CA 19-9, or location of tumor between the three groups. Lower rectal cancer showed a

Table 1. Preoperative clinical stage and postoperative pathologic stage

\begin{tabular}{lccccc}
\hline & CRG & yp stage I & yp stage II & yp stage III & Total \\
\hline Clinical stage II & 6 & 6 & 15 & 8 & 35 \\
Clinical stage III & 2 & 10 & 15 & 17 & 44 \\
Total & 8 & 16 & 30 & 25 & 79 \\
\hline
\end{tabular}

CRG, complete response group; yp, pathologic stage after neoadjuvant pretreatment.

Table 2. Clinical characteristics of 79 patients among three groups

\begin{tabular}{lcccc}
\hline Characteristics & CRG $(n=8)$ & PRG $(n=31)$ & NRG $(n=40)$ & P-value \\
\hline Age $(\mathrm{yr})$ & $63.4 \pm 6.0$ & $59.6 \pm 7.6$ & $57.3 \pm 9.7$ & 0.166 \\
$\geq 65$ & $4(50.0)$ & $8(25.8)$ & $10(25.0)$ & 0.276 \\
$\quad<65$ & $4(50.0)$ & $23(74.2)$ & $30(75.0)$ & \\
Sex $(M: F)$ & $5: 3$ & $23: 8$ & $30: 10$ & 0.567 \\
BMI $\left(\mathrm{kg} / \mathrm{m}^{2}\right)$ & $24.6 \pm 3.4$ & $23.0 \pm 3.1$ & $23.3 \pm 3.1$ & 0.484 \\
CEA & $3.4 \pm 4.1$ & $2.7 \pm 3.6$ & $3.0 \pm 4.3$ & 0.897 \\
CA19-9 & $11.5 \pm 6.9$ & $14.1 \pm 10.4$ & $15.7 \pm 16.4$ & 0.737 \\
Tumor location & & & & 0.059 \\
Lower rectum & $8(100)$ & $26(83.9)$ & $29(72.5)$ & \\
$\quad$ Middle rectum & 0 & $5(16.1)$ & $11(27.5)$ & \\
Tumor size $(\mathrm{cm})$ & 0 & 2.4 & 3.3 & 0.000 \\
\hline
\end{tabular}

Values are presented as mean \pm standard deviation or number (\%).

$C R G$, complete response group; PRG, partial response group; NRG, non-response group; $M$, male; $F$, female; BMI, body mass index; CEA, carcinoembryonic antigen. 
tendency of CR after neoadjuvant CCRT compared to mid rectal cancer $(\mathrm{P}=0.059)$. In this study, patients with lower-third rectal cancer $(5 \mathrm{~cm}$ or under from the anal verge) showed a tendency towards

Table 3. Postoperative outcomes of rectal cancer patients

\begin{tabular}{lcccc}
\hline Characteristics & CRG $(\mathrm{n}=8)$ & PRG $(\mathrm{n}=31)$ & NRG $(\mathrm{n}=40)$ & P-value \\
\hline $\begin{array}{l}\text { Type of operation } \\
\text { Low anterior resection }\end{array}$ & $7(87.5)$ & $28(90.3)$ & $36(90.0)$ & 0.894 \\
Miles' operation & $1(12.5)$ & $3(9.7)$ & $4(10.0)$ & \\
Operation time (min) & $265.6 \pm 132.6$ & $251.7 \pm 80.9$ & $253.9 \pm 121.1$ & 0.949 \\
Postoperative hospital & $12.3 \pm 5.7$ & $12.5 \pm 6.0$ & $14.5 \pm 7.2$ & 0.406 \\
stays & & & & \\
No. of lymph node & $6.9 \pm 5.5$ & $11.1 \pm 8.5$ & $13.1 \pm 7.2$ & 0.128 \\
retrieved & & & & \\
Circumferential resection & & & & 0.491 \\
margin & & & & \\
Positive & 0 & $1(3.2)$ & $2(5.0)$ & \\
Negative & $8(100)$ & $30(96.8)$ & $38(95.0)$ & \\
Postoperative complication & & & & \\
Ileus & 0 & $3(9.7)$ & $5(12.5)$ & 0.328 \\
Bleeding & 0 & 0 & $2(5.0)$ & 0.204 \\
Leakagea & 0 & 0 & $2(5.6)$ & 0.204 \\
Local recurrence & & & & - \\
Yes & 0 & $2(6.5)$ & $2(5.0)$ & \\
No & $8(100)$ & $29(93.5)$ & $38(95.0)$ & \\
Systemic recurrence & 0 & $2(6.5)$ & $15(37.5)$ & - \\
Lung & 0 & $1(3.2)$ & $6(15.0)^{b)}$ & \\
Liver & 0 & $1(3.2)$ & $8(20.0)^{b)}$ & \\
The others & 0 & 0 & $2(5.0)$ & \\
\hline
\end{tabular}

Values are presented as number (\%) or mean \pm standard deviation. CRG, complete response group; PRG, partial response group; NRG, non-response group.

${ }^{\text {a) Among }} 36$ patients. ${ }^{\text {b) }}$ ne patient had metastases in lung and liver.

Table 4. Pathological characteristics of 79 patients after neoadjuvant therapy

\begin{tabular}{|c|c|c|c|c|}
\hline Characteristics & CRG $(n=8)$ & PRG $(n=31)$ & NRG $(n=40)$ & P-value \\
\hline Clinical stage & & & & 0.340 \\
\hline$\|$ & $6(75.0)$ & $6(19.4)$ & $23(57.5)$ & \\
\hline III & $2(25.0)$ & 25 (80.6) & $17(42.5)$ & \\
\hline Differentiation & & & & 0.400 \\
\hline Well & $4(50.0)$ & $9(29.0)$ & $10(25.0)$ & \\
\hline Moderate & $3(37.5)$ & 22 (71.0) & $30(75.0)$ & \\
\hline Poor & $1(12.5)$ & 0 & 0 & \\
\hline Lymphovascular invasion & & & & 0.136 \\
\hline Positive & 0 & $3(9.7)$ & $7(17.5)$ & \\
\hline Negative & $8(100)$ & $28(90.3)$ & $33(82.5)$ & \\
\hline Neural invasion & & & & 0.291 \\
\hline Positive & 0 & 1 (3.2) & $3(7.5)$ & \\
\hline Negative & $8(100)$ & $30(96.8)$ & $37(92.5)$ & \\
\hline
\end{tabular}

CRG, complete response group; PRG, partial response group; NRG, non-response group. better response to neoadjuvant chemoradiation (CRG, 100\%; PRG, 83.9\%; NRG, 72.5\%) $(\mathrm{P}=0.059)$.

Postoperative and pathologic outcomes are listed in Tables 3 and 4. Among the 79 patients, a sphincter-saving procedure was performed in 71 cases (89.9\%). Sphincter-preserving procedures were performed in seven (87.5\%), 28 (90.3\%), and 36 patients (90\%) in the CRG, PRG, and NRG, respectively. There was no significant difference in sphincter-saving surgery. There were no differences between groups in operation time, postoperative hospital stays, harvested lymph nodes, status of circumferential resection margin and postoperative complications. Of the 71 patients who underwent sphincter-preserving procedures, there were no anastomotic leakage in the CRG and PRG, whereas there were two cases (5.6\%) in the NRG. There were no significant differences in clinical stage, pathological differentiation, lymphovascular, or neural invasion between the three groups.

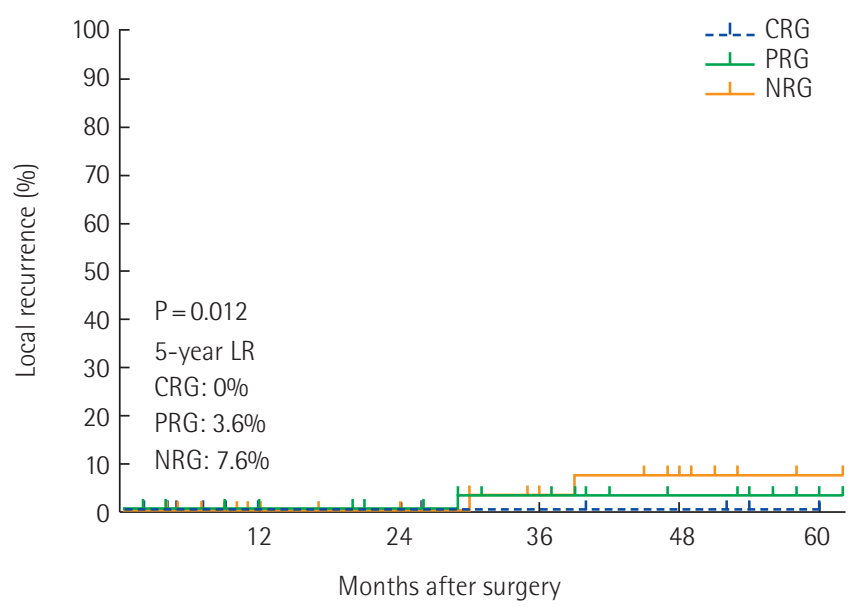

Fig. 1. The local recurrence $(L R)$ of each groups. $C R G$, complete response group; PRG, partial response group; NRG, non-response group.

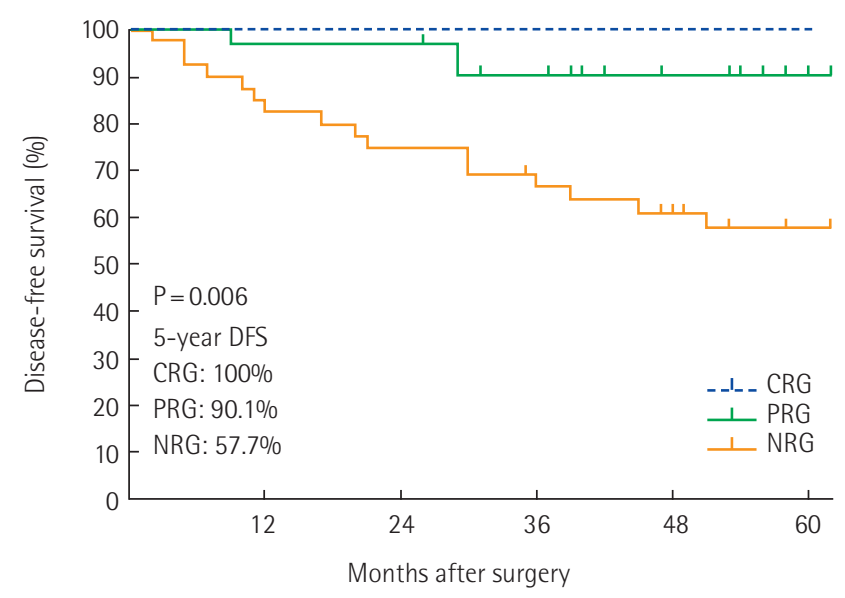

Fig. 2. The disease-free survival (DFS) of each groups. CRG, complete response group; PRG, partial response group; NRG, non-response group. 


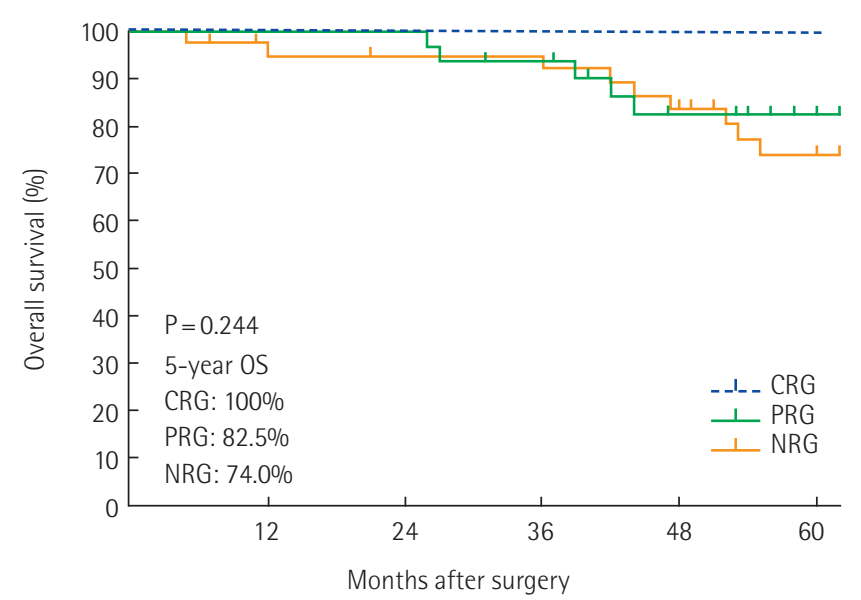

Fig. 3. The overall survival (OS) of each groups. CRG, complete response group; PRG, partial response group; NRG, non-response group.

The median follow-up of our study was 57 months (range, 3-142 months). There was a significant difference in 5-year LR (CRG, $0 \%$; PRG, 3.6\%; NRG, 7.6\%; $P=0.012$ ) (Fig. 1). The 5-year DFS rates for patients in CRG, PRG, and NRG group were $100 \%, 90.1 \%$, and $57.7 \%$, respectively $(\mathrm{P}=0.006)$ (Fig. 2 ). The 5 -year OS rates for patients in CRG, $P R G$, and NRG were $100 \%, 82.5 \%$, and $74.0 \%$, respectively $(\mathrm{P}=0.244)$ (Fig. 3). There was no significant difference in OS between the three groups.

\section{DISCUSSION}

Neoadjuvant CCRT is currently standard treatment for T3/T4 and/or node-positive rectal [2]. The benefits of chemoradiotherapy followed by radical surgery for rectal cancer are well documented, and preoperative chemoradiotherapy is more effective than postoperative chemoradiotherapy $[1,2]$.

Adjuvant chemotherapy after neoadjuvant CCRT and radical surgery for rectal cancer improves oncologic outcomes in terms of DFS and OS [12]. One of the main advantages of neoadjuvant CCRT is the possibility of achieving down-staging of the tumor, which may vary from a partial to a CR of the rectal cancer. When CR does occur, not only may it facilitate surgical R0 resection, but it may also indicate favorable tumor biology [13]. In this study, we report our long-term oncologic outcomes of locally advanced rectal cancer in terms of pathologic tumor response after neoadjuvant CCRT. Our data show that good response after neoadjuvant CCRT for rectal cancer results in good oncologic outcomes with regard to LR and DFS.

Tumor response varies from one patient to another as suggested by the three groups in this study: CRG, PRG, and NRG. In this study, pathologic results showed complete remission in eight pa- tients (10.1\%) and down-staging in 39 patients (49.4\%) out of a total of 79 patients. The results of the German Rectal Cancer Study showed an 8\% CR rate for rectal cancer after preoperative CCRT. $\mathrm{CR}$ rate has been reported as high as $27 \%$ of patients after neoadjuvant CCRT in locally advanced rectal cancer [14-16]. A meta-analysis has shown the CR rate ranged from 10.4 to $25.6 \%$ (median CR rate, $15.6 \%$ ) in an overall median follow-up of 37 months [17].

The studies reporting relatively high CR rates included relatively low stages of disease before CCRT. In our study, 44 of 79 patients (55.7\%) were stage III, which might have made our CR rate seem relatively low. There have also been efforts to improve the CR rate in advanced rectal cancer. Recently, a phase II trial adding mFOLFOX6 after neoadjuvant CRT in locally advanced rectal cancer was published [18]. In that study, the rate of CR was reported up to $38 \%$ in the group of six cycles of mFOLFOX6 between chemoradiation and surgery. Lengthening the neoadjuvant treatment with active chemoagent, such as oxaliplatin, and a longer chemoradiation-to-surgery interval provides a high rate of CR in advanced rectal cancer. Eisterer et al. [19] performed neoadjuvant chemotherapy with bevacizumab, capecitabine, and oxaliplatin followed by concomitant standard chemoradiotherapy for high risk locally advanced rectal cancer and achieved a $25 \% \mathrm{CR}$ rate.

When CR is attained, the R0 resectability of rectal cancer and anal-sphincter preservation rate increases and oncologic outcomes also improved [10,17,20,21]. Therefore, tumor response after neoadjuvant CCRT provides a predictor for oncologic outcomes and quality of life of patients in locally advanced rectal cancer. In our study, neoadjuvant CCRT did not affect the anal-sphincter preservation in rectal cancer $(\mathrm{P}=0.894)$. We speculate that this may be a consequence of a high portion of lower rectal cancer in CRG and PRG compared to NRG in spite of the statistical insignificance in the location of the rectal cancer $(\mathrm{P}=0.059)$ and the small-scale of this study.

At a median follow-up of 37 months, a meta-analysis which divided patients into two groups (CR vs. partial or no response) showed improved local and distal control as well as better OS and DFS [17]. These pooled data showed the definite advantage of CR in terms of distant recurrence and survival. A European multicenter large-scale study evaluated 566 rectal cancer patients with CR reporting lower recurrence and high survival rates [22]. In more stratified studies based on the tumor response after neoadjuvant CCRT, the patients with partial response of the tumor had a low rate of recurrence and improved survival rate than those who had no tumor response [23-25]. With a median follow-up of 44 months of 297 rectal cancer patients, Guillem et al. [24] reported that 10 -year OS was $58 \%$ and 10 -year recurrence-free survival (RFS) was $62 \%$. They also documented that a pathologic response greater than $95 \%$ in the pathologic specimen were independently 
predictive of improved long-term OS and RFS. Adjuvant chemotherapy following CCRT and radical surgery to treat locally advanced rectal cancer improved the DFS and OS [12]. Mohiuddin et al. [26] reported the effect of down-staging of the tumor after radical rectal surgery with neoadjuvant CCRT in terms of LR. Even though LR is influenced by surgical completeness associated with a negative circumferential resection margin and enough distal margin after TME, down-staging may allow higher rates of negative resection margins [27]. The response to neoadjuvant CCRT is a prognostic factor of LR and DFS. In this study, the median follow-up period was 57 months. There were significant differences in LR and 5-year DFS rates for patients in CRG, PRG, and NRG. This study shows that the response to neoadjuvant CCRT is associated with low LR and DFS. However, no significant difference was shown in 5-year OS between the three groups. This is compatible with other previous studies $[4,7,28,29]$.

The advantage of this study is in its clarification of the response to neoadjuvant CCRT for advanced rectal cancer as being related to LR and DFS directly. Although better prognoses were observed in the CRG and PRG than in the NRG in our analysis, these findings need to be interpreted carefully because of the potential inaccuracy of preoperative staging. It is important to determine the clinical stage of rectal cancer preoperatively for the adoption of neoadjuvant therapy. The accuracy of evaluating the clinical T/N stage of rectal cancer using APCT, rectal ultrasound, and rectal MRI has some limitations in these diagnostic modalities. The accuracy of an MRI in rectal cancer was reported as 55\% to $85 \%$ in diagnosing tumor depth and $47 \%$ to $89.9 \%$ in diagnosing nodal metastasis. We had the limitation of considering the preoperative clinical stage as a prognostic factor. However, a large-scale study showed that the survival in rectal cancer patients having undergone neoadjuvant treatment was determined by posttherapy pathologic stage, regardless of pretherapy clinical stage [30].

There are some limitations of this study derived from the retrospective study design. First, it was not a prospective randomized trial, our study cannot eliminate potential selection bias. The patients were not selected with definitive criteria, but were appointed to each group based on surgeon preference. And the size of the study group is small and this study is single-center study. Second, the regimen of CCRT is not homogenous. Generally, 5-fluorouracil is backbone antitumor agent, but the combination of regimen such as oxaliplatin is not equal to all patients. The change of chemotherapy regimen may influence the survival and recurrence rate. Third, the study cannot reflect degree of response. Although postoperative pathologic stage and $\mathrm{T}$ stage is used in practice generally, there is tumor regression grade (TRG) to evaluate the degree of improvement microscopically [21]. Some studies suggest TRG such as Mandard and Dworak/Rodel system is better than pathologic stage for patients with gastrointestinal cancer including rectal cancer. The TRGs are based on the ratio between residual tumor cell and remodeling fibrous reaction. But pathologic department in my hospital has not offered the TRG evaluation at the beginning period. And other prognostic factors in rectal cancer treatment such as the circumferential resection margin or mesorectal grade could not be included in this study because those factors was not thoroughly examined in all enrolled patients.

In conclusion, the treatment of locally advanced rectal cancer with neoadjuvant CCRT and radical rectal resection with TME currently provides the optimal treatment standard for a long-term oncologic outcome. Neoadjuvant CCRT can lower the tumor stage in selected rectal cancer patients and provide better DFS and lower LR.

\section{CONFLICT OF INTEREST}

No potential conflict of interest relevant to this article was reported.

\section{REFERENCES}

1. Sauer R, Becker H, Hohenberger W, Rodel C, Wittekind C, Fietkau $\mathrm{R}$, et al. Preoperative versus postoperative chemoradiotherapy for rectal cancer. N Engl J Med 2004;351:1731-40.

2. Sauer R, Liersch T, Merkel S, Fietkau R, Hohenberger W, Hess C, et al. Preoperative versus postoperative chemoradiotherapy for locally advanced rectal cancer: results of the German CAO/ARO/AIO94 randomized phase III trial after a median follow-up of 11 years. JClin Oncol 2012;30:1926-33.

3. Allal AS, Bieri S, Pelloni A, Spataro V, Anchisi S, Ambrosetti P, et al. Sphincter-sparing surgery after preoperative radiotherapy for low rectal cancers: feasibility, oncologic results and quality of life outcomes. Br J Cancer 2000;82:1131-7.

4. Quah HM, Chou JF, Gonen M, Shia J, Schrag D, Saltz LB, et al. Pathologic stage is most prognostic of disease-free survival in locally advanced rectal cancer patients after preoperative chemoradiation. Cancer 2008;113:57-64.

5. Arredondo J, Baixauli J, Beorlegui C, Arbea L, Rodriguez J, Sola JJ, et al. Prognosis factors for recurrence in patients with locally advanced rectal cancer preoperatively treated with chemoradiotherapy and adjuvant chemotherapy. Dis Colon Rectum 2013;56:416-21.

6. Fokas E, Liersch T, Fietkau R, Hohenberger W, Hess C, Becker H, et al. Downstage migration after neoadjuvant chemoradiotherapy for rectal cancer: the reverse of the Will Rogers phenomenon? Cancer 2015;121:1724-7.

7. Xu L, Cai S, Xiao T, Chen Y, Qiu H, Wu B, et al. Prognostic significance of tumour regression grade after neoadjuvant chemoradio- 
therapy for a cohort of patients with locally advanced rectal cancer: an 8-year retrospective single-institutional study. Colorectal Dis 2017;19:O263-71.

8. Jalil O, Claydon L, Arulampalam T. Review of neoadjuvant chemotherapy alone in locally advanced rectal cancer. J Gastrointest Cancer 2015;46:219-36.

9. Bouzourene H, Bosman FT, Seelentag W, Matter M, Coucke P. Importance of tumor regression assessment in predicting the outcome in patients with locally advanced rectal carcinoma who are treated with preoperative radiotherapy. Cancer 2002;94:1121-30.

10. Maas M, Nelemans PJ, Valentini V, Das P, Rodel C, Kuo LJ, et al. Long-term outcome in patients with a pathological complete response after chemoradiation for rectal cancer: a pooled analysis of individual patient data. Lancet Oncol 2010;11:835-44.

11. Kim DY, Joo JK, Park YK, Ryu SY, Kim YJ, Kim SK, et al. Is palliative resection necessary for gastric carcinoma patients? Langenbecks Arch Surg 2008;393:31-5.

12. Petrelli F, Coinu A, Lonati V, Barni S. A systematic review and meta-analysis of adjuvant chemotherapy after neoadjuvant treatment and surgery for rectal cancer. Int J Colorectal Dis 2015;30:447-57.

13. Crane CH, Skibber JM, Feig BW, Vauthey JN, Thames HD, Curley $\mathrm{SA}$, et al. Response to preoperative chemoradiation increases the use of sphincter-preserving surgery in patients with locally advanced low rectal carcinoma. Cancer 2003;97:517-24.

14. Hartley A, Ho KF, McConkey C, Geh JI. Pathological complete response following pre-operative chemoradiotherapy in rectal cancer: analysis of phase II/III trials. Br J Radiol 2005;78:934-8.

15. Pucciarelli S, Toppan P, Friso ML, Russo V, Pasetto L, Urso E, et al. Complete pathologic response following preoperative chemoradiation therapy for middle to lower rectal cancer is not a prognostic factor for a better outcome. Dis Colon Rectum 2004;47:1798-807.

16. Uzcudun AE, Batlle JF, Velasco JC, Sanchez Santos ME, Carpeno Jde C, Grande AG, et al. Efficacy of preoperative radiation therapy for resectable rectal adenocarcinoma when combined with oral tegafur-uracil modulated with leucovorin: results from a phase II study. Dis Colon Rectum 2002;45:1349-58.

17. Zorcolo L, Rosman AS, Restivo A, Pisano M, Nigri GR, Fancellu A, et al. Complete pathologic response after combined modality treatment for rectal cancer and long-term survival: a meta-analysis. Ann Surg Oncol 2012;19:2822-32.

18. Garcia-Aguilar J, Chow OS, Smith DD, Marcet JE, Cataldo PA, Varma MG, et al. Effect of adding mFOLFOX6 after neoadjuvant chemoradiation in locally advanced rectal cancer: a multicentre, phase 2 trial. Lancet Oncol 2015;16:957-66.

19. Eisterer W, Piringer G, De Vries A, Ofner D, Greil R, Tschmelitsch J, et al. Neoadjuvant chemotherapy with capecitabine, oxaliplatin and bevacizumab followed by concomitant chemoradiation and surgical resection in locally advanced rectal cancer with high risk of recurrence: a phase ii study. Anticancer Res 2017;37:2683-91.

20. Garcia-Aguilar J, Hernandez de Anda E, Sirivongs P, Lee SH, Madoff RD, Rothenberger DA. A pathologic complete response to preoperative chemoradiation is associated with lower local recurrence and improved survival in rectal cancer patients treated by mesorectal excision. Dis Colon Rectum 2003;46:298-304.

21. Martin ST, Heneghan HM, Winter DC. Systematic review and meta-analysis of outcomes following pathological complete response to neoadjuvant chemoradiotherapy for rectal cancer. Br J Surg 2012; 99:918-28.

22. Capirci C, Valentini V, Cionini L, De Paoli A, Rodel C, GlynneJones R, et al. Prognostic value of pathologic complete response after neoadjuvant therapy in locally advanced rectal cancer: longterm analysis of 566 ypCR patients. Int J Radiat Oncol Biol Phys 2008;72:99-107.

23. Rodel C, Martus P, Papadoupolos T, Fuzesi L, Klimpfinger M, Fietkau R, et al. Prognostic significance of tumor regression after preoperative chemoradiotherapy for rectal cancer. J Clin Oncol 2005;23:8688-96.

24. Guillem JG, Chessin DB, Cohen AM, Shia J, Mazumdar M, Enker $\mathrm{W}$, et al. Long-term oncologic outcome following preoperative combined modality therapy and total mesorectal excision of locally advanced rectal cancer. Ann Surg 2005;241:829-36.

25. Biondo S, Navarro M, Marti-Rague J, Arriola E, Pares D, Del Rio C, et al. Response to neoadjuvant therapy for rectal cancer: influence on long-term results. Colorectal Dis 2005;7:472-9.

26. Mohiuddin M, Marks J, Marks G. Management of rectal cancer: short- vs. long-course preoperative radiation. Int J Radiat Oncol Biol Phys 2008; $72: 636-43$.

27. Dworak O, Keilholz L, Hoffmann A. Pathological features of rectal cancer after preoperative radiochemotherapy. Int J Colorectal Dis 1997;12:19-23.

28. Valentini V, Coco C, Picciocchi A, Morganti AG, Trodella L, Ciabattoni A, et al. Does downstaging predict improved outcome after preoperative chemoradiation for extraperitoneal locally advanced rectal cancer? A long-term analysis of 165 patients. Int J Radiat Oncol Biol Phys 2002;53:664-74.

29. Roh MS, Colangelo LH, O'Connell MJ, Yothers G, Deutsch M, Allegra CJ, et al. Preoperative multimodality therapy improves disease-free survival in patients with carcinoma of the rectum: NSABP R-03. J Clin Oncol 2009;27:5124-30.

30. Delitto D, George TJ Jr, Loftus TJ, Qiu P, Chang GJ, Allegra CJ, et al. Prognostic value of clinical vs pathologic stage in rectal cancer patients receiving neoadjuvant therapy. J Natl Cancer Inst 2018;110: 460-6. 